\title{
Eukaryotic algae: where lies the diversity of oxygenic photosynthesis
}

\author{
Pierre Cardol · Fabrice Franck
}

Published online: 13 August 2010

(C) Springer Science+Business Media B.V. 2010

\section{Introduction}

Due to their fast growth, homogeneity as cell populations and easy handling, microalgae attracted plant biologists as laboratory organisms for the study of the metabolism and physiology of photosynthetic cells. This led, for example, to the extensive use of the green alga Chlamydomonas reinhardtii for studying photosynthesis, to such a degree that this alga was nicknamed the green yeast (e.g. Goodenough 1992). Reinforcing the dominant position of Chlamydomonas, the availability of its nuclear genome sequence (Merchant et al. 2007) made also possible the identification of a minimal set of proteins (designated the GreenCut) that were likely involved specifically in chloroplast function within the green lineage. Recent advances in approaching the functions of these proteins are highlighted in this special issue (Grossman et al. 2010). Many pioneering works and more recent findings on the regulation of photosynthetic electron transport arose from studies on eukaryotic microalgae, such as chlororespiration (Bennoun 1982) and cyclic electron flow (Iwai et al. 2010) in Chlamydomonas, or state transitions in the green alga Chlorella pyrenoidosa (Bonaventura and Meyers 1969). Recent developments concerned with state transitions and auxiliary electron transfer pathways are reviewed in this

\section{P. Cardol ( $\square)$}

Laboratory of Genetics of Microorganisms, Department of Life Sciences, Institute of Plant Biology, B22, University of Liège, 27 Boulevard du Rectorat, 4000 Liège, Belgium

e-mail: pierre.cardol@ulg.ac.be

\section{F. Franck}

Laboratory of Plant Biochemistry and Photobiology, Department of Life Sciences, Institute of Plant Biology, B22, University of Liège, 27 Boulevard du Rectorat, 4000 Liège, Belgium issue (Alric 2010; Lemeille and Rochaix 2010; Peltier et al. 2010).

Oxygenic photosynthesis in eukaryotes is not restricted to terrestrial plants and plant-model algal systems (mainly green algae). Indeed photosynthesis in eukaryotic cell was acquired laterally through a primary endosymbiotic event with a cyanobacteria and this gave rise to plants, green algae, red algae and glaucophytes (e.g. Rodriguez-Ezpeleta et al. 2005). As examples, two contributions to this issue highlight the unique architecture of the photosynthetic apparatus in red algae (Neilson and Durnford 2010; Su et al. 2010). Photosynthesis then spread throughout different eukaryotic kingdoms laterally via secondary endosymbiosis, most commonly through the engulfment by a nonphotosynthetic host of a red alga or green alga, giving rise for example to diatoms and euglena, respectively (e.g. Archibald 2009). Among eukaryotic algae, diatoms play a considerable role in the primary productivity of oceans and thus in biogeochemical carbon cycle, comparable to that of cyanobacteria. The acquisition of these so-called secondary plastids also accounts for much of the photosynthetic diversity on the planet, i.e. it was associated with a variety of adaptation strategies involving the photosynthetic process. Some of these peculiarities are dealt with here in reviews on carotenoid biosynthesis in diatoms (Bertrand 2010), light-harvesting processes (Neilson and Durnford 2010), photoprotective mechanisms (Goss and Jakob 2010), and inorganic carbon acquisition (Raven 2010).

At a time when human societies are facing major challenges in terms of climate control, renewable energy production, and nutrition of populations across the planet, the understanding of photosynthetic processes and their features in different groups of algae forms a basis for the development of algal biotechnology. The availability of suitable algal strains and the optimization of the mass 
culture process are two crucial issues if one wants to consider the use of large-scale algal cultures for high-yield production of biomass, whatever its use. In this issue, review articles pay tribute to the importance of the use of microalgae with respect to the production of biomass (Grobbelaar 2010), hydrogen (Ghysels and Franck 2010) or secondary carotenoids (Lemoine and Schoefs 2010).

Finally, the availability of techniques that allow the in vivo study of photosynthesis is an equally relevant aspect for evaluating photosynthetic performances in batch culture and for exploring fundamental aspects of photosynthetic regulation in the various lineages. Two contributions to this issue highlight significant technical advances (Alric 2010; Bailleul et al. 2010).

\section{References}

Alric J (2010) Cyclic electron flow around photosystem I in unicellular green algae. Photosynth Res. doi:10.1007/s11120010-9566-4

Archibald JM (2009) The puzzle of plastid evolution. Curr Biol 19:R81-R88

Bailleul B, Cardol P, Breyton C, Finazzi G (2010) Electrochromism: a useful probe to study algal photosynthesis. Photosynth Res. doi: 10.1007/s11120-010-9579-z

Bennoun P (1982) A respiratory chain in the thylakoid membranes of Chlamydomonas reinhardtii. Prog Clin Biol Res 102b:291-298

Bertrand M (2010) Carotenoid biosynthesis in diatoms. Photosynth Res. doi:10.1007/s11120-010-9589-x

Bonaventura C, Meyers J (1969) Fluorescence and oxygen evolution from Chlorella pyrenoidosa. Biochim Biophys Acta 189:366-383

Ghysels B, Franck F (2010) Hydrogen photo-evolution upon S-deprivation stepwise: an illustration of microalgal photosynthetic and metabolic flexibility and a step stone for future biotechnological methods of renewable $\mathrm{H} 2$ production. Photosynth Res. doi:10.1007/s11120-010-9582-4
Goodenough UW (1992) Green yeast. Cell 70:533-538

Goss R, Jakob T (2010) Regulation and function of xanthophyll cycle-dependent photoprotection in algae. Photosynth Res. doi: 10.1007/s11120-010-9536-x

Grobbelaar J (2010) Microalgal biomass production: challenges and realities. Photosynth Res. doi:10.1007/s11120-010-9573-5

Grossman A, Karpowicz SJ, Heinnickel M, Dewez D, Hamel B, Dent $\mathrm{R}$ et al (2010) Phylogenomic analysis of the Chlamydomonas genome unmasks proteins potentially involved in photosynthetic function and regulation. Photosynth Res. doi:10.1007/s11120010-9555-7

Iwai M, Takizawa K, Tokutsu R, Okamuro A, Takahashi Y, Minagawa $J$ (2010) Isolation of the elusive supercomplex that drives cyclic electron flow in photosynthesis. Nature 464: $1210-1213$

Lemeille S, Rochaix JD (2010) State transitions at the crossroad of thylakoid signalling pathways. Photosynth Res. doi:10.1007/s 11120-010-9538-8

Lemoine Y, Schoefs B (2010) Secondary ketocarotenoid biosynthesis in algae: a multifunctional response to stress. Photosynth Res. doi:10.1007/s11120-010-9583-3

Merchant SS, Prochnik SE, Vallon O, Harris EH, Karpowicz SJ, Witman GB et al (2007) The Chlamydomonas genome reveals the evolution of key animal and plant functions. Science 318: 245-250

Neilson JA, Durnford DG (2010) Structural and functional diversification of the light-harvesting complexes in photosynthetic eukaryotes. Photosynth Res. doi:10.1007/s11120-010-9576-2

Peltier G, Tolleter D, Billon E, Cournac L (2010) Auxiliary electron transport pathways in chloroplasts of microalgae. Photosynth. Res. doi 10.1007/s11120-010-9575-3

Raven JA (2010) Inorganic carbon acquisition by eukaryotic algae: four current questions Photosynth. Res. doi 10.1007/s11120010-9563-7

Rodriguez-Ezpeleta N, Brinkmann H, Burey SC, Roure B, Burger G, Loffelhardt W et al (2005) Monophyly of primary photosynthetic eukaryotes: green plants, red algae, and glaucophytes. Curr Biol 15:1325-1330

Su HN, Xie BB, Zhang XY, Zhou BC, Zhang YZ (2010) The supramolecular architecture, function, and regulation of thylakoid membranes in red algae: an overview. Photosynth. Res. doi 10.1007/s11120-010-9560-x 